\title{
Auger at the Telescope Array: toward a direct cross-calibration of surface-detector stations
}

\section{S. Quinn ${ }^{* 1}$ for the Pierre Auger Collaboration ${ }^{a}$ and Telescope Array Collaboration ${ }^{b}$; S. Colognes ${ }^{2}$, B. Courty ${ }^{2}$, B. Genolini ${ }^{3}$, L. Guglielmi ${ }^{2}$, P. Lebrun ${ }^{4}$, M. Marton ${ }^{5}$, E. Rauly ${ }^{3}, \mathbf{T}$. Trung ${ }^{3}$, O. Wolf ${ }^{6}$}

${ }^{1}$ Dept. of Physics, Case Western Reserve Univ., Cleveland, Ohio, USA

${ }^{2}$ Laboratoire Astroparticules et Cosmologie (APC), Univ. Paris 7, CNRS-IN2P3, Paris, France

${ }^{3}$ Institut de Physique Nucléaire d'Orsay (IPNO), Univ. Paris 11, CNRS-IN2P3, Orsay, France

${ }^{4}$ Fermilab, Batavia IL, USA

${ }^{5}$ Laboratoire de Physique Subatomique et de Cosmologie (LPSC), Univ. Joseph Fourier, INPG, CNRS-IN2P3, Grenoble, France

${ }^{6}$ Physics Department, Colorado School of Mines, Golden CO, USA

${ }^{a}$ Observatorio Pierre Auger, Av. San Martín Norte 304, 5613 Malargüe, Argentina

E-mail: auger_spokespersons@fnal.gov

Full author list: auger.org/archive/authors_icrc_2017.html

${ }^{b}$ Telescope Array Project, 201 James Fletcher Bldg., 115 S. 1400 East, Salt Lake City, UT

84112-0830, USA

E-mail: ta-icrc@cosmic.utah.edu

Full author list: telescopearray.org/index.php/research/collaborators

Since 2007 the Telescope Array Project (TA) and Pierre Auger Observatory (Auger) have collected extensive data sets spanning several orders of magnitude of the cosmic-ray spectrum. In both experiments the majority of data is generated from the surface-detector (SD) array as a result of its very high duty cycle. These data are then calibrated for energy with fluorescence detectors using a hybrid approach. The TA and Auger experiments use different SD station designs, giving them different sensitivities to extensive air-shower components. We seek to understand and cross-validate these complementary detectors on a hardware level. In this paper we present an update on the progress of this in-situ cross-calibration program. Presently two Auger waterCherenkov detectors and two TA scintillator stations are co-located at the TA central laser facility (CLF). We review the hardware enabling the readout of these detectors for high-energy events. Additionally, we show expanded calibration data sets of minimum-ionizing particle (MIP) versus vertical-equivalent muon (VEM) responses, along with preliminary results for the Auger doublet. A sample event reconstruction displaying observed Auger and TA signals is also presented.

35th International Cosmic Ray Conference - ICRC2017

10-20 July, 2017

Bexco, Busan, Korea

* Speaker. 


\section{Introduction}

The Pierre Auger Observatory is a hybrid cosmic-ray (CR) experiment located in Mendoza, Argentina, which detects extensive air showers (EAS) using four fluorescence-telescope detectors and 1660 surface-detector (SD) stations [1]. Fluorescence observations provide high-quality data of shower energy as well as depth of maximum shower development, important for composition studies, but have a limited duty cycle. The subset of hybrid events passing stringent quality cuts is used to calibrate "SD only" events which account for the majority of reconstructed EAS data.

The Telescope Array Project is also a hybrid CR observatory located in central Utah, USA [2]. In addition to its three fluorescence-telescope detector stations, TA operates 507 SD stations, with $\sim 400$ more detectors being introduced over the next few years to quadruple the detection area [3]. Both experiments utilize SD stations with self-contained electronics, communications, and solar power systems. The detection medium in Auger is used to count air-shower muons but is also sensitive to the electromagnetic shower component, while TA detectors count ionizing particles indiscriminately.

The Auger SD station is a water-Cherenkov detector (WCD). Relativistic leptons and highenergy photons generate signals via Cherenkov radiation and pair production, respectively, which are collected by photomultiplier tubes (PMTs). The Telescope Array SD station uses plastic scintillator panels. Fluorescent scintillation light is collected by wavelength-shifting fibers and guided to PMTs in a dual layer setup.

To improve understanding of the energy spectrum and origin of ultrahigh-energy cosmic rays (UHECRs), the TA and Auger Collaborations have performed analyses of a joint data set $[4,5]$. These studies benefit from larger statistics and full sky coverage. A recent analysis [5] concluded that UHECR composition results of both experiments agree within systematic uncertainties. The energy spectra also agree within systematic errors up to the ankle, but diverge toward the highest energies $(E \gtrsim 50 \mathrm{EeV})$ near the Greisen, Zatsepin, Kuz'min (GZK) cutoff [6, 7]. The source of this discrepancy remains to be fully explained, but possibilities might include: the result of experimental effects or different astrophysics scenarios in the northern and southern skies. We are investigating the possibility of energy-dependent experimental effects using a direct comparison of surface-detection methods through a two-phase joint cross-calibration program. Phase I, where data for station-level responses to the same air shower is compared, has been underway for roughly 8 months. We review results from earlier work [8,9], provide an overview of hardware deployed and prototypes in development, and present an updated cross calibration curve along with an example shower reconstruction.

\section{Detectors in the field}

For phase I we are currently collecting data from: one Auger south (AS) WCD, one prototype Auger north (AN) WCD and two TA stations (see Figure 1). The AN and AS doublet is formed to study the response between the conventional AS station (used at the Pierre Auger Observatory) and prototype AN station.

The AS and AN detectors use a $3.6 \mathrm{~m}$ diameter, $1.2 \mathrm{~m}$ high, reflectively lined (Tyvek $($ ) ) tank filled with 12,000 L of purified water. In AS three symmetrically distributed PMTs, each $1.2 \mathrm{~m}$ 
from the central axis [1], are used. Low-gain (AC coupled anode) and high-gain (8th dynode) channels for each PMT are input to the electronics and digitized by 10-bit $40 \mathrm{MHz}$ semi-flash ADCs. These traces are analyzed and time-stamped (GPS synchronized clock) by a programmable logic device running triggering firmware. A data-acquisition (DAQ) system running on a single board computer (SBC) (see section 3.1) evaluates the timestamps of station-level (T2) events. Coincident time-stamps in a certain geometry surrounding the central laser facility (CLF) generate a physics trigger-the SBC requests the stations to transfer ADC traces for the event. Online calibration data from the previous minute for AS, and the previous 14 minutes for AN, are also retrieved to convert each ADC waveform to an integrated vertical muon-equivalent signal (VEM).

The AN station uses a single, central, downward facing 9" PMT. The electronics are similar to AS, but use fewer components since only one PMT is digitized. The key difference is a new 10-bit $100-\mathrm{MHz}$ flash ADC (FADC) which processes four channels for increased dynamic range as well as a new Linux based operating system (Debian) which includes many convenient applications. The anode signal is split into $0.1 \times, 1 \times$, and $30 \times$ channels. Instead of the 8 th dynode, the 5 th stage is used to achieve a larger overall dynamic range.

The TA station uses two layers of polyvinyl toluene scintillators $3 \mathrm{~m}^{2}$ in area and $1.2 \mathrm{~cm}$ thick. In each layer, light is guided through 104 wavelength shifting fibers to a $30 \mathrm{~mm}$ PMT. The anode is digitized by 12 -bit 50-MHz FADCs which are processed by triggers implemented on field-programmable gate arrays (FPGA) and transmitted as station-level (L1) events. A hierarchical triggering system is implemented based on minimum-ionizing particles (MIP). Station-level events are communicated wirelessly to a central acquisition computer. Physics triggers are similar to those in the Auger array, requiring a coincidence of geometrical and temporal station-level events.

The four detectors are co-located at the CLF site. The Auger doublet is located in the northeast corner, one TA station is in the northwest and the other in the southwest corner. The maximum separation between stations is $\approx 44 \mathrm{~m}$ from the Auger doublet to the southwest TA station.

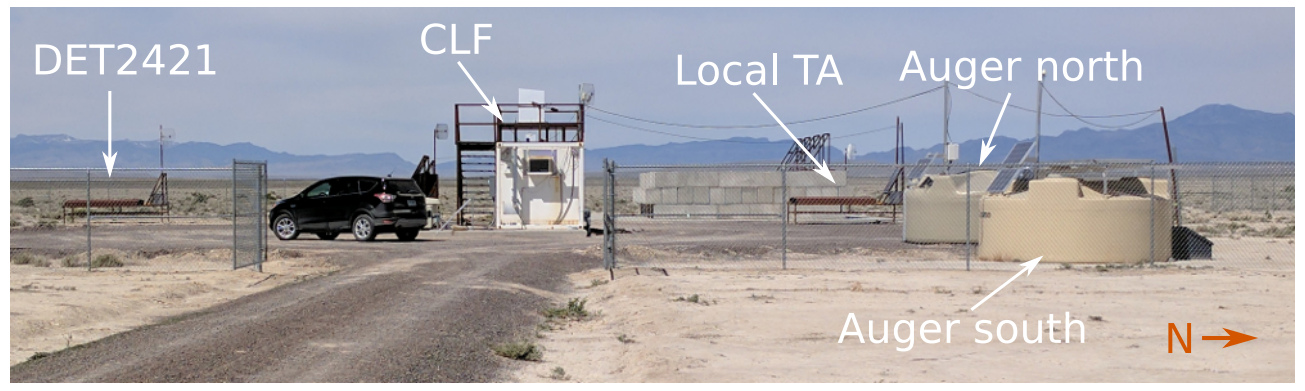

Figure 1: Photo of hardware deployed at the TA CLF site. Custom cabling was setup along guy wires from stations to the CLF for data acquisition. North is to the right of the page.

\section{DAQ setup}

\subsection{High-level description}

To retrieve data from the Auger stations, which are designed to operate wirelessly, we modified the electronics to communicate over a physical wire connected to a SBC housed inside the CLF. An 
external trigger is required to read out the Auger doublet for low-energy events which is provided by a primitive threshold-comparator circuit attached to a bare (without electronics) TA SD station: this is known as the "local trigger". For a direct comparison between Auger and TA waveforms, a second fully functional TA station was installed in the southwest corner and is able to send data for physics events (higher energy showers) using the standard TA radio protocol.

\subsection{TA global (physics) trigger}

During March 2016 a TA SD electronics kit was brought online inside the CLF container. It uses a parabolic radio antenna to listen for physics triggers sent to the TA SD array. The firmware was modified to relay time-stamp information for shower candidates observed by the TA DAQ computer and is forwarded over a RS-232 serial connection to the SBC inside the CLF.

The southwest "global" TA station (DET2421 in Figure 1) uses standard TA electronics and operates in normal TA acquisition mode. The L1 time stamps from this detector are ignored by the TA DAQ computer-hence this station does not participate with adjacent stations to contribute toward the physics/array trigger. However, when an event is observed it transmits data normally. The relevant traces for this project are only collected when a core lands in a constrained area of CLF neighboring stations. Auger traces are also retrieved for the global trigger timestamp, so a direct comparison of station waveforms is possible. With the appropriate conversion between MIP and VEM, it is also possible to insert Auger data into a lateral distribution function (LDF) to optionally perform a dual detector reconstruction.

\subsection{CLF vicinity "local" trigger}

The northwest TA station, installed August 2016, is used to investigate showers of low and intermediate-energy with cores close to the CLF. Currently it only operates as an external trigger for the Auger doublet until a way to collect the TA waveforms over a wired connection is devised. It is roughly $32 \mathrm{~m}$ from the doublet. The PMTs are operated nominally at $-1.2 \mathrm{kV}$, and when the anode output of both simultaneously cross a threshold set at $<-92 \mathrm{mV}$ the circuit transmits a logic pulse over a RG58 cable to a development FPGA board (MicroZed) running time-tagging firmware referenced to GPS time. The signal is time-stamped and sent over a serial connection to the Auger SBC (Raspberry Pi 2 Model B). This station is operated at a base rate of $\approx 3-15 \mathrm{~Hz}$.

\subsection{Trigger decision hardware and software}

Custom interconnects and cabling bring AN CANbus and AS RS-232 serial data into the CLF in place of radios. A radio-protocol emulation program on the SBC is used to decode AS data and send control or read out commands to the AS station, while similar software handles AN communication. These packages provide real-time station-level event lists (T2 triggers). These lists, combined with another program which parses the CLF local and global triggers, are analyzed for coincidences. A read out request (T3 trigger) is sent if the time difference between the Auger doublet event and global event is $<100 \mu \mathrm{s}$, or if the local trigger and Auger doublet difference is $<20 \mu$ s. A high-level diagram of the setup is displayed in Figure 2. Auger north can optionally be configured to save its data to a local disk. 
Events are archived by day (UTC) and uploaded to remote servers where data decompression and analysis are performed using code written for this project. Monitoring and detector performance information is updated daily.
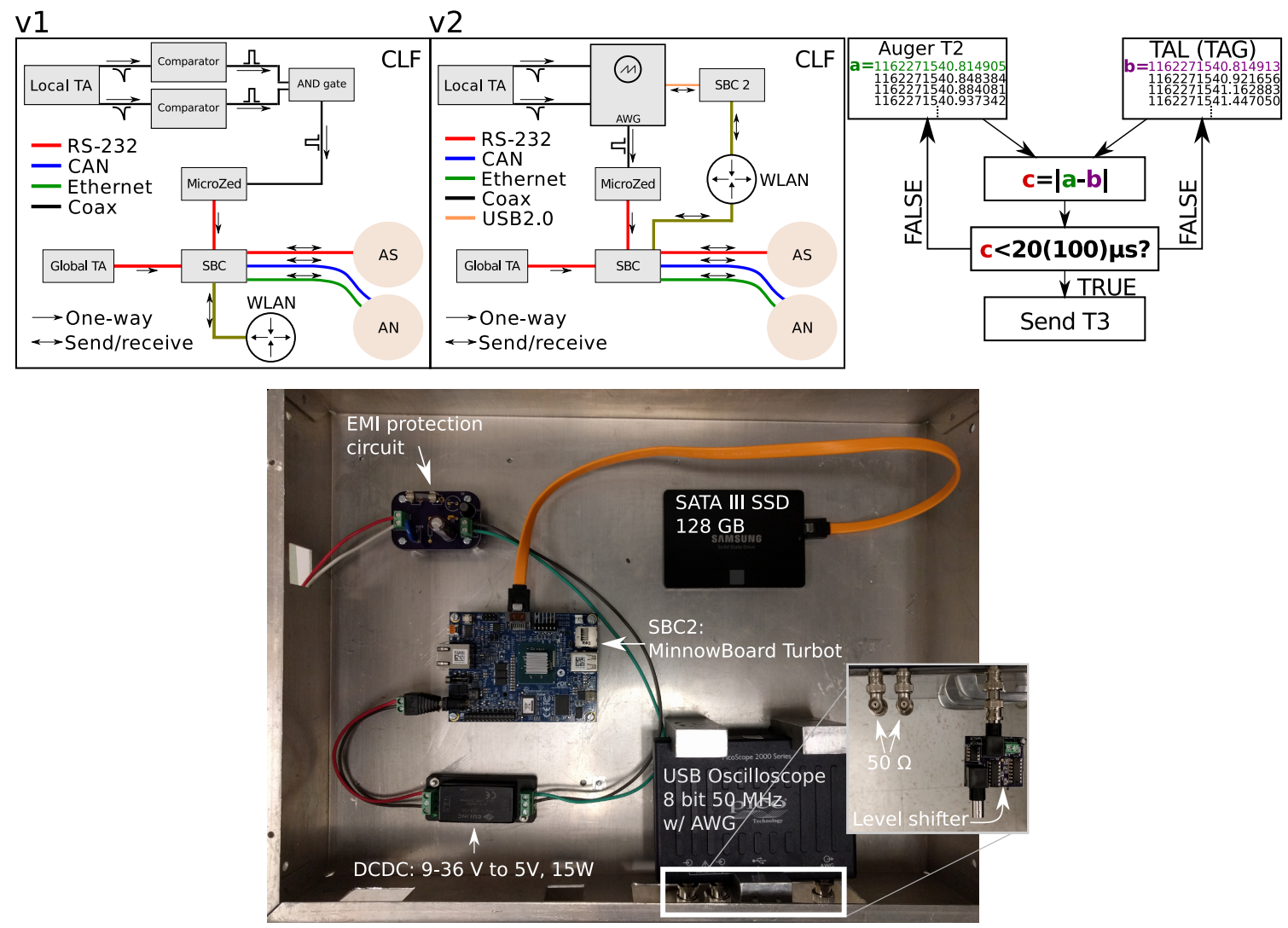

Figure 2: Top left: block diagram of current local trigger hardware deployed at the CLF site (see text for details). Top middle: future local trigger setup under development. Top right: trigger-condition flow chart showing the "do while" loop to compare example local time stamps. Items in parentheses refer to the global trigger. Bottom: photo of the future prototype local trigger hardware to be installed in the field.

\subsubsection{Prototype advanced local trigger design}

The current local trigger design uses a simple threshold comparator circuit which measures the peak amplitude of PMT waveforms. This results in a bias favoring inclined "old" showers dominated by muons, compared to vertical "young" showers where the signal is spread out in time and contains a larger fraction of electromagnetic components. Also, the current setup doesn't provide any information about the waveform morphology initiating the trigger.

To address this limitation we have developed a new prototype local trigger system which uses a Pico Technology 2206B USB oscilloscope connected to a Minnowboard Turbot SBC. Using the Pico software, we can remotely configure programmable triggers to fire on temporal features in addition to threshold levels. This setup offers a closer approximation to Auger and TA station level triggers in lieu of TA electronics for the local detector. The new SBC is equipped with a hard drive so calibration data and event waveforms can be stored. The associated time stamp is relayed from 
the SBC inside the CLF over the local network. The prototype will be a drop-in replacement for the current circuit—a diagram and photo of the design is shown in Figure 2.

\section{Analysis \& Results}

\subsection{Auger north and south comparison}

The Auger doublet is used to study the performance of AN and AS observations for the same EAS. From November 2016 through May 2017 we have recorded 1635 coincidences generated by the external local trigger. We show logarithmized data in Figure 3, along with a fit. No quality cuts or VEM thresholds have been applied. We find a least squares best fit power law of $S_{A S}=$ $1.18\left(S_{A N}\right)^{0.92}$. The ideal expectation is $S_{A S}=S_{A N}$, however it must be stressed our calibration source is low energy showers with small cores and potentially highly variable particle densities. It is not possible to do any post selection with our current setup-Poissonian fluctuations are included. We will continue to investigate this correlation. To test the responses using a more constrained input, we look at signal correlations for global events where both stations have data. For this comparison we find a best fit powerlaw of $S_{A S}=0.76\left(S_{A N}\right)^{1.06}$.
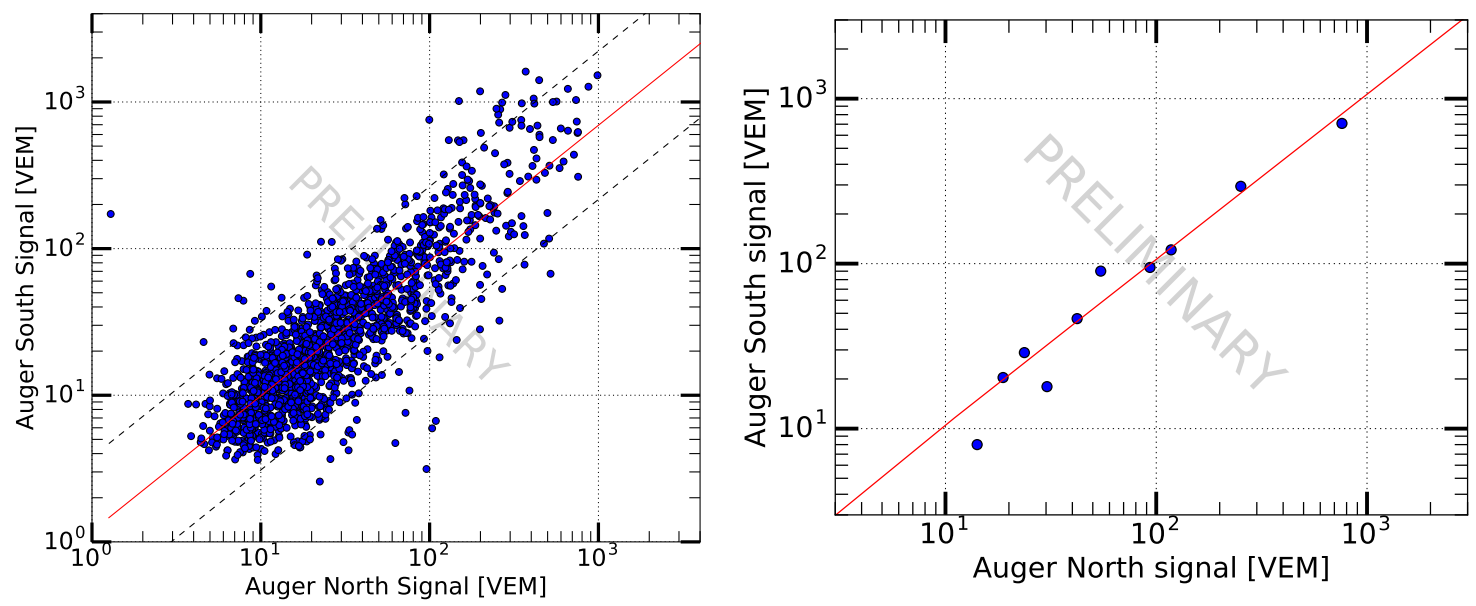

Figure 3: Left: Auger south and Auger north signal pairs for local events. Solid red line is the least squares fit and dashed black lines are the $95 \%$ prediction interval. Right: Auger south and Auger north signal pairs for global events only. Best fit line is solid red.

\subsection{Global event reconstruction and trace comparison}

We have collected 54 global coincidences between the AS and neighboring TA stations up to May 2017. In Figure 4 we show FADC waveforms and core position for a single event within 2 $\mathrm{km}$ from the CLF. This is one of the 8 events in the data set which passes standard TA SD quality cuts outlined in [10]. From a TA reconstruction we find the following observables: $E=4.58 \mathrm{EeV}$, $(\theta, \varphi)=(38.28,216.69)^{\circ}$ and $r=0.82 \mathrm{~km}$, where $E,(\theta, \varphi)$ and $r$ are primary energy, zenith \& azimuth angles and core distance, respectively. Using the standard calibration techniques, we find average integrated signals of 36.58 MIP and 56.50 VEM. In addition to a comparison of integrated signals, we can also compare waveform shapes, but we reserve this for a future study. 

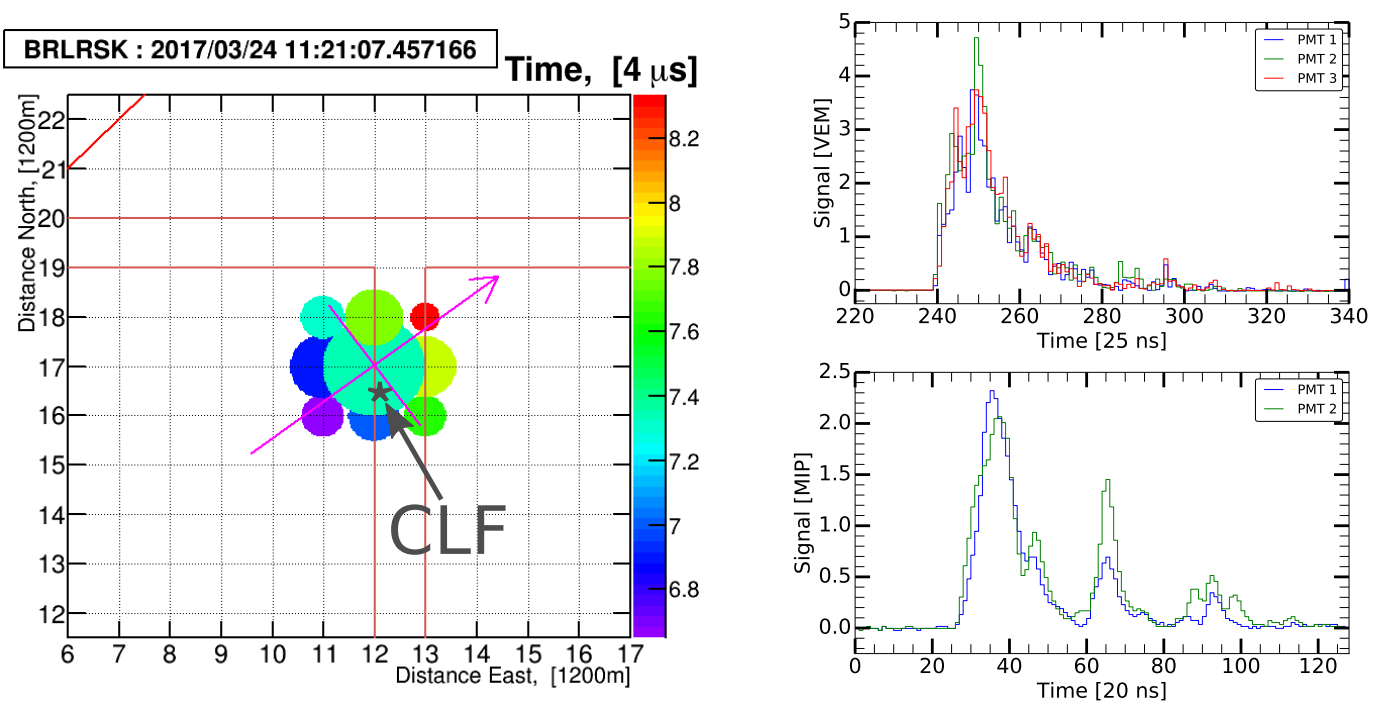

Figure 4: Left: position and timing information for a global event also seen by TA and Auger detectors co-located at CLF. Top right: Auger south high gain waveforms. Bottom right: DET2421 waveforms.

\subsection{MIP vs. VEM cross-calibration curve and simulations}

The global event data can be used to form a cross calibration curve for MIP and VEM signals, see Figure 5. With more statistics this curve can be used to study detector responses to the same shower, and, further, how any potential difference in detector sensitivity depends on air-shower parameters. For phase II this curve can be used to translate between MIP and VEM signals.

We present an initial result for such a study using simulations in the Auger $\overline{\text { Off }}\lfloor$ line framework [1] with newly implemented scintillator detectors [11] on top of a WCD. To start, an ad-hoc Monte Carlo (MC) shower population was generated with the following parameter space: $E=3.98 \mathrm{EeV}$, $\theta=\{0,12,25,36,45,53\}^{\circ}, r=600 \mathrm{~m}$. The Auger scintillator has an area of $4 \mathrm{~m}^{2}$ while a TA SD is $3 \mathrm{~m}^{2}$ : the integrated TA MIP values will roughly scale as 0.75 of the Auger scintillator MIP values. We show these simulations and a recently updated MIP vs. VEM plot in Figure 5. For the current data set, DET2421 and Auger signal responses and by extension TA reconstruction parameters, appear to be consistent with these simulations. More detailed event-by-event shower and reconstruction simulations are underway.

\section{Conclusion}

In this work we have highlighted progress made since the publication of $[8,9]$. Initial results of the Auger doublet correlation for a highly variable input population appears reasonable. The doublet data for select global events is more tightly correlated. We presented a reconstruction with corresponding waveforms for a sample event. An updated MIP-VEM cross-calibration curve was shown for all raw data as well as events which pass a standard quality cut. These data were compared to MC simulations using a prototype Auger upgrade detector for a variety of shower parameters-while preliminary, the data mostly agree with these simulations. Initial designs to improve the local trigger system were proposed, and phase II was briefly motivated. 

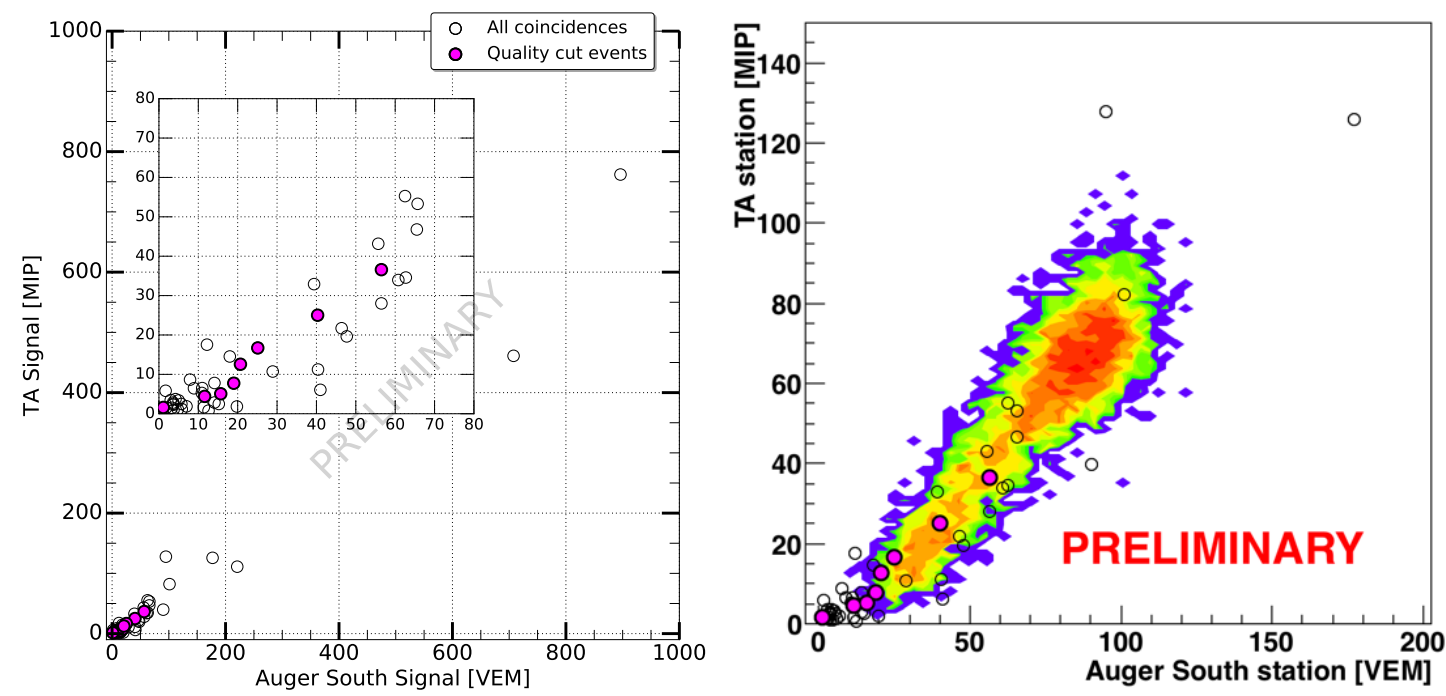

Figure 5: Left: cross-calibration curve for most recent data set. Open black circles include all global trigger data while filled magenta circles are events that pass quality cuts [10]. Inset plot is zoomed in area of signals with < 80 VEM/MIP. Right: Similar to left inset, but with MC simulation contours overlaid.

\section{Acknowledgements}

This project was supported in part by JSPS Grant-in-Aid for Scientific Research (A) 25247035.

\section{References}

[1] The Pierre Auger Collaboration, Nucl. Instr. Meth. Phys. Res. A 798, 172 (2015)

[2] The Telescope Array Collaboration, Nucl. Instr. Meth. Phys. Res. A 689, 87 (2012)

[3] H. Sagawa for the Telescope Array Collaboration, Proc. of the 34th Int. Cosmic Ray Conf., The Hague, The Netherlands, PoS(ICRC2015)657 (2015)

[4] O. Deligny for the Pierre Auger and Telescope Array Collaborations, Proc. of the 34th Int. Cosmic Ray Conf., The Hague, The Netherlands, PoS(ICRC2015)395 (2015)

[5] M. Unger for the Pierre Auger and Telescope Array Collaborations, Proc. of the 34th Int. Cosmic Ray Conf., The Hague, The Netherlands, PoS(ICRC2015)307 (2015)

[6] K. Greisen, Phys. Rev. Lett. 16, 748 (1966)

[7] G. T. Zatsepin, V. A. Kuz'min, JETPL 4, $78 Z$ (1966)

[8] R. Takeishi for the Pierre Auger and Telescope Array Collaborations, Proc. of the 34th Int. Cosmic Ray Conf., The Hague, The Netherlands, PoS(ICRC2015)393 (2015)

[9] S. Quinn for the Pierre Auger and Telescope Array Collaborations, Proc. of Int. Symposium for UHECRs, Kyoto, Japan. Submitted for publication (2017)

[10] The Telescope Array Collaboration, ApJ 768, L1 (2013)

[11] The Pierre Auger Collaboration, The Pierre Auger Observatory Upgrade - Preliminary Design Report, arXiv:1604.03637 (2016) 